\title{
On generalizations of two curious divisibility properties
}

\author{
Aynur Yalçiner
}




\title{
ON GENERALIZATIONS OF TWO CURIOUS DIVISIBILITY PROPERTIES
}

\author{
AYNUR YALÇINER
}

Received 18 September, 2012

\begin{abstract}
In this paper, we extend two curious divisibility properties for the general second order linear recurrence $\left\{U_{n}(p, q)\right\}$. We also give new recursive identities for the general second linear recurrences $\left\{U_{n}(p, q)\right\}$ and $\left\{V_{n}(p, q)\right\}$. These results generalize the results given by E. Kilıç, "A matrix approach for generalizing two curious divisibility properties", Miskolc Math. Notes, vol. 13., No. 2, pp. 389-396, 2012.
\end{abstract}

2010 Mathematics Subject Classification: 11B37; 15B36

Keywords: binary linear recursion, divisibility property, matrix method

\section{INTRODUCTION}

Let $p$ and $q$ be nonzero integers such that $p^{2}+4 q \neq 0$. For $n>1$, the generalized Fibonacci sequence $\left\{U_{n}(p, q)\right\}$ and the generalized Lucas sequence $\left\{V_{n}(p, q)\right\}$ are defined by

$$
U_{n}(p, q)=p U_{n-1}(p, q)+q U_{n-2}(p, q)
$$

and

$$
V_{n}(p, q)=p V_{n-1}(p, q)+q V_{n-2}(p, q),
$$

where $U_{0}(p, q)=0, U_{1}(p, q)=1$ and $V_{0}(p, q)=2, V_{1}(p, q)=p$, respectively.

Let $\alpha$ and $\beta$ be the roots of the equation $x^{2}-p x-q=0$. Then the Binet formulas of the sequences $\left\{U_{n}(p, q)\right\}$ and $\left\{V_{n}(p, q)\right\}$ are given by

$$
U_{n}(p, q)=\frac{\alpha^{n}-\beta^{n}}{\alpha-\beta} \text { and } V_{n}(p, q)=\alpha^{n}+\beta^{n}
$$

If $p=q=1$, then $U_{n}(1,1)=F_{n}$ ( $n$th Fibonacci number) and $V_{n}(1,1)=L_{n}(n$th Lucas number).

It is a well known fact that

$$
\operatorname{gcd}\left(F_{n}, F_{m}\right)=F_{\operatorname{gcd}(n, m)} .
$$

It is also known that $F_{k n}$ is a multiple of $F_{n}$, for all integers $k$ and $n$. In [9], the author showed that, for $n>2$, the Fibonacci number $F_{m}$ is a multiply of $F_{n}^{2}$ if and only if 
$m$ is multiply of $n F_{n}$ (for more details see [4]). Also, in [1], the author obtained the following divisibility properties:

i) $F_{k n-1}-F_{n-1}^{k}$ is divisible by $F_{n}^{2}$,

ii) $F_{k n-2}-(-1)^{k+1} F_{n-2}^{k}$ is divisible by $F_{n}^{2}$,

where $n, k \geq 1$. Recently Kılıç [7] generalized these results for a general second order linear recursion $\left\{U_{n}(p, 1)\right\}$ as follows:

$$
U_{r}^{k-1}(p, 1) U_{k n-r}(p, 1)-(-1)^{(r-1)(k+1)} U_{n-r}^{k}(p, 1) \text { is divisible by } U_{n}^{2}(p, 1) .
$$

Furthermore, the author found new recursive identities for the general second order linear recurrences $\left\{U_{n}(p, 1)\right\}$ and $\left\{V_{n}(p, 1)\right\}$.

In this paper, for the case $q \neq 1$, we show that

$U_{r}^{k-1}(p, q) U_{k n-r}(p, q)-(-1)^{(r-1)(k+1)} q^{r(k-1)} U_{n-r}^{k}(p, q)$ is divisible by $U_{n}^{2}(p, q)$.

To do that we use matrix methods. Matrix methods are useful tools for derivating some properties of linear recurrences (see $[3,5,6,8,10]$ ). We consider the quotient

$$
\frac{U_{r}^{k-1} U_{k n-r}-(-1)^{(r-1)(k+1)} q^{r(k-1)} U_{n-r}^{k}}{U_{n}^{2}},
$$

where $n, k \geq 1$. We define a generating matrix for this quotient for fixed $n$ and increasing values of $k$. Then we give an explicit statement for the quotient. Also, by considering this explicit statement, we find new recursive identities for the general second order linear recurrences $\left\{U_{n}(p, q)\right\}$ and $\left\{V_{n}(p, q)\right\}$. Thus we obtain a generalization of the results given in [7].

Throughout this study, for simplicity, we will denote $U_{n}(p, q)$ by $U_{n}$ and $V_{n}(p, q)$ by $V_{n}$.

\section{MAin Results}

Before we give our main results, we need some auxiliary results and definitions. Denote the quotient $\left(U_{r}^{k-1} U_{k n-r}-(-1)^{(r-1)(k+1)} q^{r(k-1)} U_{n-r}^{k}\right) / U_{n}^{2}$ by $s(n, k)$. Define two matrices $H(n)$ and $G(n, k)$ of order 3 as follows:

$$
H(n)=\left[\begin{array}{ccc}
A_{n-1} & B_{n} & -(-q)^{n+r} U_{r}^{2} U_{n-r} \\
1 & 0 & 0 \\
0 & 1 & 0
\end{array}\right]
$$

and

$$
G(n, k)=\left[\begin{array}{ccc}
s(n, k+2) & t(n, k+2) & -(-q)^{n+r} U_{r}^{2} U_{n-r} s(n, k+1) \\
s(n, k+1) & t(n, k+1) & -(-q)^{n+r} U_{r}^{2} U_{n-r} s(n, k) \\
s(n, k) & t(n, k) & -(-q)^{n+r} U_{r}^{2} U_{n-r} s(n, k-1)
\end{array}\right],
$$

where

$$
A_{n-1}=U_{r} V_{n}-(-q)^{r} U_{n-r},
$$




$$
B_{n}=(-q)^{r} U_{r} V_{n} U_{n-r}-(-q)^{n} U_{r}^{2}
$$

and

$$
\begin{aligned}
t(n, k) & =\left((-q)^{r} U_{r}^{k-1} U_{k n} U_{n-r}^{2}+(-1)^{r-1} q^{2 n-r} U_{r}^{k+1} U_{n(k-2)}\right. \\
& \left.+(-1)^{(r+1)(k-1)} q^{r(k-1)} U_{r} U_{2 n} U_{n-r}^{k}\right) / U_{n}^{3}
\end{aligned}
$$

Lemma 1. For $n \geq 1$, the eigenvalues of $H(n)$ are $U_{r} \alpha^{n}, U_{r} \beta^{n}$ and $-(-q)^{r} U_{n-r}$.

Proof. The characteristic polynomial of $H(n)$ is

$$
x^{3}-A_{n-1} x^{2}-B_{n} x+(-q)^{n+r} U_{r}^{2} U_{n-r}=0
$$

and it is factorized as

$$
\left(x-U_{r} \alpha^{n}\right)\left(x-U_{r} \beta^{n}\right)\left(x+(-q)^{r} U_{n-r}\right)=0,
$$

as required.

Thus the first main result of this paper is the following.

Theorem 1. For $n>1$,

$$
H(n)^{k}=G(n, k) .
$$

Proof. In the proof, we will use induction on $k$. Since $G(n, 1)=H(n)$, the result is true when $k=1$. Now assume that $H(n)^{k-1}=G(n, k-1)$. Then, by the definitions of $s(n, k)$ and $t(n, k)$, we have

$$
A_{n-1} s(n, k+1)+t(n, k+1)=s(n, k+2)
$$

and

$$
B_{n} s(n, k+1)-(-q)^{n+r} U_{r}^{2} U_{n-r} s(n, k)=t(n, k+2) .
$$

This completes the proof.

As a consequence of this theorem, we can see that the matrix $H(n)$ generate the $s(n, k)$. Since the elements of $H(n)$ are integers, the quotient $s(n, k)$ are integers.

Also from Theorem 1 in [2], we have the following result for the combinatorial representation of $s(n, k)$.

\section{Corollary 1.}

$$
s(n, k)=\sum_{\left(l_{1}, l_{2}, l_{3}\right)}\left(\begin{array}{c}
l_{1}+l_{2}+l_{3} \\
l_{1}, l_{2}, l_{3}
\end{array}\right)(-1)^{(n+r-1) l_{3}} A_{n-1}^{l_{1}} B_{n}^{l_{2}} U_{r}^{2 l_{3}} U_{n-r}^{l_{3}},
$$

where the summation is over nonnegative integers satisfying $l_{1}+2 l_{2}+3 l_{3}=k-2$.

As another main result, we have the following theorem. 
Theorem 2. For $n, k \geq 1$

$$
\begin{aligned}
(G(n, k))_{3,1} & =s(n, k)= \\
& =\frac{(-q)^{n-r} U_{r}^{k} U_{n(k-1)}+(-1)^{k}(-q)^{r(k-1)} U_{n} U_{n-r}^{k}+U_{r}^{k-1} U_{k n} U_{n-r}}{U_{n}^{3}} .
\end{aligned}
$$

Proof. Since the eigenvalues of $H(n)$ are distinct, $H(n)$ is diagonalizable as

$$
V^{-1} H(n) V=D,
$$

where

$$
V=\left[\begin{array}{ccc}
U_{r}^{2} \alpha^{2 n} & U_{r}^{2} \beta^{2 n} & q^{2 r} U_{n-r}^{2} \\
U_{r} \alpha^{n} & U_{r} \beta^{n} & -(-q)^{r} U_{n-r} \\
1 & 1 & 1
\end{array}\right],
$$

and $D=\operatorname{diag}\left(U_{r} \alpha^{n}, U_{r} \beta^{n},-(-q)^{r} U_{n-r}\right)$. Therefore, we obtain $V^{-1} H(n)^{k} V=$ $D^{k}$. By Theorem 1, we write $V^{-1} G(n, k) V=D^{k}$. Then we have the following linear equation system:

$$
\begin{aligned}
g_{i 1} U_{r}^{2} \alpha^{2 n}+g_{i 2} U_{r} \alpha^{n}+g_{i 3} & =U^{k+(3-i)} \alpha^{k n+(3-i) n} \\
g_{i 1} U_{r}^{2} \beta^{2 n}+g_{i 2} U_{r} \beta^{n}+g_{i 3} & =U^{k+(3-i)} \beta^{k n+(3-i) n} \\
g_{i 1} q^{2 r} U_{n-r}^{2}-g_{i 2}(-q)^{r} U_{n-r}+g_{i 3} & =(-1)^{(r-1)(k+3-i)} q^{k r+(3-i) r} U_{n-r}^{k+(3-i)}
\end{aligned}
$$

Using the identities

and

$$
U_{n-r} U_{n+r}-U_{n}^{2}=-(-q)^{n-r} U_{r}^{2}
$$

$$
q^{r} U_{n-r}+(-1)^{r} U_{r} V_{n}=(-1)^{r} U_{n+r},
$$

the solution of the above linear equation system gives the claimed result.

By considering definition of $s(n, k)$, we have the following consequence of Theorem 2.

Corollary 2. Let $n, k$ and $r$ arbitrary integers. Then

$$
U_{n-r} U_{k n}=U_{n} U_{k n-r}-(-q)^{n-r} U_{r} U_{n(k-1)} .
$$

The next result presents a similar expression by considering generalized Lucas sequence $\left\{V_{n}\right\}$.

Theorem 3. For all integers $n, k, r$,

$$
U_{n-r} V_{k n}=U_{n} V_{k n-r}-(-q)^{n-r} U_{r} V_{n(k-1)} .
$$

Proof. Using Binet formulas of the sequence $\left\{U_{n}\right\}$ and $\left\{V_{n}\right\}$, we have

$$
\begin{aligned}
& U_{n} V_{k n-r}-(-q)^{n-r} U_{r} V_{n(k-1)}= \\
& =\left(\alpha^{k n+n-r}-\beta^{k n+n-r}+\alpha^{n} \beta^{k n-r}-\alpha^{k n-r} \beta^{n}-(-q)^{n-r} \alpha^{k n-n+r}\right. \\
& \left.+(-q)^{n-r} \beta^{k n-n+r}-(-q)^{n-r} \alpha^{r} \beta^{k n-n}+(-q)^{n-r} \alpha^{k n-n} \beta^{r}\right) /(\alpha-\beta)
\end{aligned}
$$




$$
\begin{aligned}
& =\left(\alpha^{k n+n-r}-\beta^{k n+n-r}-(-q)^{n-r} \alpha^{k n-n+r}+(-q)^{n-r} \beta^{k n-n+r}\right) /(\alpha-\beta) \\
& =\left(\alpha^{n-r}-\beta^{n-r}\right)\left(\alpha^{k n}+\beta^{k n}\right) /(\alpha-\beta) \\
& =U_{n-r} V_{k n} .
\end{aligned}
$$

The proof is complete.

\section{ACKNOWLEDGEMENT}

The author would like to thank the referee for constructive comments, which improved the presentation of the paper. This work is supported by the Scientific Research Projects Office (BAP) of Selcuk University.

\section{REFERENCES}

[1] M. Cavachi, "Some properties of the terms of the Fibonacci sequence," Gaz. Mat., Bucur., vol. 85, pp. 290-293, 1980.

[2] W. Y. Chen and J. D. Louck, "The combinatorial power of the companion matrix," Linear Algebra Appl., vol. 232, pp. 261-278, 1996.

[3] M. C. Er, "Sums of Fibonacci numbers by matrix methods," Fibonacci Q., vol. 22, pp. 204-207, 1984.

[4] R. L. Graham, D. E. Knuth, and O. Patashnik, Concrete mathematics: a foundation for computer science. 2nd ed., 2nd ed. Amsterdam: Addison-Wesley Publishing Group, 1994.

[5] E. Kilic, "The generalized order- $k$ Fibonacci-Pell sequence by matrix methods," J. Comput. Appl. Math., vol. 209, no. 2, pp. 133-145, 2007.

[6] E. Kiliç, "The generalized Fibonomial matrix,” Eur. J. Comb., vol. 31, no. 1, pp. 193-209, 2010.

[7] E. Kiliç, "A matrix approach for generalizing two curious divisibility properties," Math. Notes, Miskolc, vol. 13, no. 2, pp. 389-396, 2012.

[8] E. Kiliç and P. Stănică, "A matrix approach for general higher order linear recurrences," Bull. Malays. Math. Sci. Soc. (2), vol. 34, no. 1, pp. 51-67, 2011.

[9] Y. V. Matiyasevich, "Enumerable sets are diophantine," Sov. Math., Dokl., vol. 11, pp. 354-358, 1970.

[10] R. A. Rosenbaum, "An application of matrices to linear recursion relations," Am. Math. Mon., vol. 66, pp. 792-793, 1959.

\section{Author's address}

\section{Aynur Yalçıner}

Selcuk University, Science Faculty, Department of Mathematics, 42075, Campus, Konya, Turkey

E-mail address: ayalcinereselcuk.edu.tr 